\title{
Discussion on the Use of Chinese Medicine and Institutional Innovation in Community Health Management
}

\author{
Shen Jun-long, Wang-qi, Zhang-yan, Ma Hong-yao \\ School of Economic Trade Management, Nanjing University of Chinese Medicine, Nanjing, China \\ Email address: \\ j1shen2005@126.com (Shen Jun-long)
}

\section{To cite this article:}

Shen Jun-long, Wang-qi, Zhang-yan, Ma Hong-yao. Discussion on the Use of Chinese Medicine and Institutional Innovation in Community Health Management. Science Journal of Public Health. Vol. 4, No. 3, 2016, pp. 235-240. doi: 10.11648/j.sjph.20160403.22

Received: April 21, 2016; Accepted: May 19, 2016; Published: May 23, 2016

\begin{abstract}
The eighth session of the Third Plenary put forth that social reform and innovation, institutional innovation and social governance, and community health management are an important part of social governance. With characteristics such as simple and inexpensive testing, and the service features and advantages of chronic disease prevention and control that are more fully realized in community health management, Chinese medicine is based on the ideology of Heaven and the harmony between man and nature, which is applied to the treatment of diseases, health care methods, and maintenance and improvement of the body. Thus, with the appropriate technology, Chinese medicine can be a good fit for community health management and improvement of immune function. The traditional community governance model failed to take advantage of medical services in the implementation of chronic disease prevention and control. A new economic theory, the multi-center collaborative governance theory, provides a theoretical basis for using the characteristics of Chinese medicine in community health management innovation. This new economic model integrates the community health management needs of the government and the market, fosters the participation of social organizations and residents, and creates opportunities to apply the characteristics of Chinese medicine to community health management through the Governance of Pluralism.
\end{abstract}

Keywords: Community Characteristics of Chinese Medicine, Health Management, System Innovation

\section{Introduction}

With changes in the human disease spectrum and the medical model, the accelerated process of population aging, and the increase in people's health needs, implementation of a community-based health management system has become an important public health issue in developed countries. However, owing to the delayed focus on community health care, combined with the lack of funding for community health services, the irrational allocation of health resources, the poor quality of health care services, and other such reasons, community health management is still in its infancy [1]. The concept of health proposed by the World Health Organization (WHO) has led to the transformation of the modern medical model to that of community health management. The services and technology involved in Chinese medicine do not harm human tissue because the medication prescribed are derived from natural sources, the focus is on restoring the balance in the body, and there are no toxic side effects or adverse reactions; therefore, it is very suitable for long-term community health management. To this end, as a part of the "Traditional Chinese Medicine Development Strategy Plan (2016-2030)," in February 2016, Nisshin announced that, by 2020, universal access to Chinese medicine services would be achieved, and that there was an "urgent need for inheritance, development, and optimal utilization of Chinese medicine in Health Reforms, to benefit human health."

\section{Community Health Management: The Need to Strengthen the Dissemination of Knowledge Regarding Chinese Medicine}

Traditional Chinese medicine (TCM) is essentially a healthy culture, and the theories of TCM are based on concepts such as the balance between yin and yang, five grams of health checks and balances, and Manner into each other, 
that the body and spirit can affect health, reached treating disease effect. The difference between Chinese medicine and modern medical health care services is that the former is concerned with, whereas the latter is concerned with the disease. Chinese medicine health services focus on human disease by emphasizing on disease prevention by righting to evil, which involves solving the "root" cause of the problem. The WHO pointed out that health is a state of physical, mental, and social well-being, and not merely the absence of disease or infirmity. Health is thus considered to involve three dimensions: physical health, which indicates that the physical structure is intact and functioning properly, maintaining coordination between the body and the environment; mental health, which means that people are in a good mental state, including the correct understanding of the self and the environment, and appropriate adaptation to the environment; and good social adaptability, that is, the individual's ability to play an appropriate role in the social system, such that the body facilitates the individual to effectively play a role that is compatible with his/her social status, conforms to social norms, and facilitates the harmonious integration of the individual and the society [2]. As the traditional Western medical model mainly focuses on the treatment of disease by removing its cause and eliminating pathogenic bacteria and viruses, medications often produce toxic side effects and adverse reactions. Therefore, based on the concept of health, the WHO recommended national health agencies to guide residents regarding health management.

The TCM culture and knowledge regarding disease prevention and health care provides theoretical and practical support for the utilization of community health management resources. The maximum extent possible, with the meaning of the new concept of health, the health of consumers adapt to changes in demand [3]. Chapman and Pelletier suggested that health management aims to reduce disease and to improve the health of individuals, health services, and health care usage patterns through the use of new technology initiatives and organized and focused management of cost-effective preventive methods [4]. Thus, in community health management, it is essential for professionals to conduct comprehensive monitoring, analysis, and assessment of risk factors for health intervention and provide appropriate health advice and guidance to the public [5]. In the pursuit of the greatest degree of health and happiness, community health management helps mobilize individuals, groups and social organizations, and local government initiatives for the effective use of limited resources to achieve maximum health effects [6] by spreading the popularity of Chinese medicine and culture, improving health awareness in the society, fostering healthy behaviors and lifestyles in the population, promoting every member of the society to improve their quality of life, and ultimately helping people live a happy and harmonious life.

To strengthen the Chinese medicine culture, dissemination of knowledge in the community is essential. Therefore, it is necessary to use the community health service system as the basis and make full use of government and community resources, community health education and health promotion programs, application of medical knowledge to overcome the health risk factors, and guidance and intervention [7]. Characteristic of Chinese medicine community health management is reflected in the whole population health management, requires a combination of knowledge of Chinese and Western medicine, community health center will work in the treatment of diseases from passive to active health management, the formation of prevention, combining prevention with control, in complementary medicine, reasonable co-ordination, optimal allocation, efficient use of health resources in Western medicine, Chinese medicine can save on overall expenses, long-term maintenance of physical and mental health of the people, to maximize social welfare.

\section{Community Health Management Requires an Innovative Model Based on Chinese Medicine}

Chinese medicine services and technology are characterized by "simple inexpensive tests," which proves advantageous in the promotion of community health management. Chinese medicine Medicinal and Edible, static and dynamic binding, builds character's health prevention and treatment of chronic diseases have better results. Indicators and should be combined with modern medical technology to collect, monitor community health information to Chinese medicine theory and create a healthy community through evaluation, guidance, and intervention using the new management model based on Chinese medicine.

\subsection{Promote the Use of Chinese Medicine by Doctors and Patients to Foster Mental Health and Social Harmony}

TCM, focuses on helping patients fight diseases by guiding them to love, to love love gram, and maintain a "neutral" state of mind. The State Drug Administration published the "Chinese Medicine Hospital of Traditional Chinese Culture Construction Guide" and pointed out "the core value of Chinese medicine can be summarized in two words, benevolence and refinement" [8].

Chinese medicine is based on the Confucian culture of benevolence that was followed by ancient physicians. TCM is strongly based on "benevolence" because it is considered the only way to ensure that people are cared for and respected during the use of the services and technology of Chinese medicine. Thus, benevolence indicates an extremely rich cultural value of ethics and technology integration. However, this demand for high professional ethics from medical workers burdens them and necessitates them to learn and use medical technology, and train and cultivate moral character in the service of the residents will be able to reflect the "benevolence of Medical Techniques Germany." Thus, the job requirements of a professional in Chinese medicine include "benevolence." In addition, according to Zhou Song, a language researcher, "Jing Xu move straight," with a caring heart, medical practices to "Kuoran Grand Duke." By "He" in Chinese 
medicine status "Holy degree," "Treatise on Febrile and Miscellaneous Diseases" appeared 81 times, "Plain Questions" appears 79 times, "Miraculous Pivot" appears 74 times [9]. Further, in TCM, "and" is valuable for both doctors and patients, and in medical practice, the two sides need to maintain physiological and psychological peace, and to control their thoughts and behaviors to effectively promote harmony between themselves, with the natural environment, and with the society, to finally achieve physical and mental unity and coordination. Owing to the maintenance of harmony between the yin and yang qi of the internal organs, and the blood to the body to maintain coordination of semen, the "and" status is an important physiological basis for good emotions, which are conducive to the health of human blood and semen. In the context of modern social knowledge explosion, rapid technological development, people's fast-paced life, and their need for relief from high stress, Chinese medicine has a detached secular culture based on values such as peace, the Heaven's cognitive style, and the pursuit of aesthetic taste in the realm of art. The effective dissemination and use of these values will help protect the health of community residents, such that people find psychological and spiritual harmony, and coordination between the individual and the society amidst the noisy modern social life, which is consistent with the goals of community health management.

Create a community and cultural characteristics of Chinese medicine mode in "benevolence doctors' belief guidelines, creation and context, play the advantages of Chinese medicine characteristics of technology, so Chinese medicine services in communities embodying Big Medicine sincere feelings, to protect medical workers in well serve the residents, sincere communication with the residents, all for the sake of the people, compassion Puji create a harmonious doctor-patient relationship.

\subsection{Use of the Approach of "Treating Diseases" in Chinese Medicine to Develop a Good Lifestyle for the Residents}

"Preventive Treatment of Disease" is the "The Yellow Emperor's Internal Canon of Medicine" important theoretical medicine and traditional methods of disease control in 2008, the State Pharmaceutical Administration launched the "Preventive Treatment of Disease" health project, which provided strong impetus to the development of Chinese medicine in preventive healthcare. In the book, "Introduction to Medicine," edited by Professor He Yumin, it was acknowledged that "medicine is a way of life in nature." TCM is based on the thousands of years of clinical practice and the wisdom of ancient Chinese physicians, which is the origin of all thought homologous, chosen by Dawson, the road through a philosophical wisdom of the Orient. Epistemology is its ideological foundation "World and I, and life, all things and I a." Who is a product of nature. Inevitable changes occur in the human body in line with the basic laws of nature, and the natural climate changes that occur during the four seasons have a major impact on human physiology and pathology. Community health management should guide people regarding the general concepts of Chinese medicine, such as
Heaven guidance, imitation of the nature's method for health and preventive care, following a righteous culture and technology, eliminating evil, and using methods to balance the yin and yang during treatment. Such effective communication about Chinese medicine and its technology is essential for helping residents develop healthy behaviors through good guidance and intervention [10]. Chinese medicine, "Preventive Treatment of Disease" is a disease of the body without the disease simmering, made without the intervention transfer the three states, "Preventive Treatment of Disease " take "not disease prevention," "both diseases anti change" "Chai defense complex" interference technology, with low cost and good effect characteristics, community health management involves appropriate use of diet and exercise for disease prevention, health, and healthcare, to help high-risk groups, the incidence has threatened populations, interfere with its sub-health status [11].

Thus, Chinese medicine needs to be used in community health service management to strengthen the knowledge of healthy living, for intervention related to "Preventive Treatment of Disease," and widely disseminate knowledge regarding community preventive health care, which can promote the residents' health literacy and health awareness in their daily life, and promote healthy habits lay the foundation for the protection of the health of community residents.

\subsection{Use of the Technical Services Characteristic of Chinese Medicine to Protect the Health and Safety of Community Residents}

TCM treatment methods include simple and inexpensive tests to examine a variety of non-pharmacological characteristics, thus providing unique resources for community health management. The dialectical theory of TCM treatment relies on systematic philosophical and logical perspectives on hair cut herbs. It employs diagnostic methods based on look and smell, and other such methods to collect clinical data on the patient's history, symptoms, and other comprehensive assessments of disease etiology, pathogenesis, disease location, and disease resistance to speculate disease trends. Additionally, physical differences in illness characteristics are examined to design personalized treatment programs based on local conditions. Thus, this diagnostic modality is less dependent on large equipment inspection. Further, Chinese medicine uses familiar methods such as acupuncture, moxibustion, massage, cupping, qigong, smoked, washing, dressing, paste, scraping, therapeutic herbs, etc., as well as some specialty treatments such as sunlight therapy, sand therapy, spa treatments, and other natural therapies. For mental emotional therapy it often uses provision of love or affection to promote physicochemical love, music therapy, etc. Finally, the use of tai chi, Wu Qin Xi, Ba Duan Jin, playing, writing, painting, and other healthcare interventions have also been reported [12].

Community health management based on Chinese medicine needs to utilize these advantages of Chinese medicine technology. The technical characteristics of Chinese medicine have a natural resistance and compliance, cause no harm or 
side effects and adverse reactions to the human body, and use simple and appropriate technology that are quick and easy to implement; are convenient, economical, and practical; and are popular among the masses. Thus, by providing little guidance to medical staff, appropriate and effective community health management interventions can be implemented.

As is evident in the above discussion, owing to the feasibility of services and advantages of Chinese medicine, and the theoretical and technical support it offers, medical resources can be used for community health management. However, in China, community health management is still in its early stages. Additionally, there is a shortage of community medicine and health resources, lack of experience regarding health management, and residents agree with the lower series of difficulties and problems. Chinese medicine services into the development of community health management, not only to learn from foreign experience in community health management should be based on the actual situation of Chinese medicine resources and supply capacity of the community, innovative and effective health management system with Chinese characteristics and policies to promote mechanisms for participation provide an institutional guarantee of the main building, and configuration and optimization of health resources for sustainable development.

\section{Use of Chinese Medicine for Community Health Management System Innovation}

Community characteristics of Chinese medicine with Chinese characteristics community health management, cannot copy the Western countries mode, we need to manage institutional innovation. The application of Chinese medicine for community health management system innovation requires theoretical support. The new system of modern theory provides us the theoretical basis for this process. Central meaning the system is from the Latin verb instituere (founded or established) derived, and it shows in the form of Activity on identified, or a combination of the structure. With reference to new institutionalism, a system is seen as a series of rules, norms, and organizations. "A system involves the development of a series of rules, moral ethics, and compliance behaviors intended to pursue the welfare or interests of individuals by maximizing healthcare utilization." Further, "a system provides a framework for human interaction, and constitutes the establishment of a community, or more precisely, an economic order of cooperation and competition."

\subsection{Characteristics of Chinese Medicine That Support Community Health Service Management Product Innovation}

According to Samuelson's theory, social products are classified as private and public goods, where public goods have non-competitive and non-exclusive features. Public goods can be divided into pure public goods and quasi-public goods, where the former are non-competitive and non-exclusive, involve non-profit investment, and should be fully provided by the government, such as the defense, public safety, public health, and so on [13]. Quasi-public goods have features of pure public goods and private goods, as they are not entirely exclusive or competitive, and consumption spillover benefits are based on the nature of public as were as private demand. Some operational, education, health, and other such services fall under quasi-public goods [14]. Chinese medicine community health management services are quasi-public goods that are governed by the government, social organizations, and corporations, which work together to make innovative products and services available to community residents.

\subsection{Institutional Innovation in Chinese Medicine Community Health Management}

The failure of the government to provide public goods has always been difficult to address. With the emergence of the Western concept of welfare state, such a "welfare crisis" with reference to the provision of public goods in the 1970s was greatly criticized. Thus, owing to the "market failure" and "government failure" with reference to the provision of public goods, the traditional resource allocation mode or the "market-government" dichotomous model did not meet the needs of public affairs governance [15].

Under the negative list management system and decentralization of government power shrink does not mean weakening the government's responsibility, but the adjustment function. Therefore, the Government withdrew from some competitive areas to concentrate its limited resources and energy on fundamental and strategic affairs. For instance, in areas such as the use of mechanism design theory for system design, the use of an incentive compatible mechanism to design community governance systems, and the use of the characteristics of Chinese medicine to develop a community health management system, the government's main tasks are as follows: (1) to play a strategic role in guiding the development of Chinese medicine community health management through the development of appropriate policies, planning, standards and guidelines for other players to assume and fulfill monitoring and evaluation functions, incentives and regulatory acts for the parties involved, and favorable environment for the equitable participation of the body to mobilize the enthusiasm of various stakeholders; (2) to promote propaganda and guidance by using modern mass media to popularize the health and medicine culture, to promote health education, health consciousness, and people's understanding of medical knowledge to improve the health of the masses through Chinese medicine literacy; and (3) to strengthen the leading role of investment in community health management, increase security and financial investment, promote the construction of basic hardware facilities for community health services, develop standardized training programs on Chinese medicine for general practitioners, and set-up community health centers to oversee the maintenance of community health records, disease surveillance, cancer screening, health assessment, and other infrastructure work. 
Proponents of the system school, Elinor and Vincent Ostrom proposed the "polycentric governance" theory [16], a new public management theory that advocated many representative theories [17]. The core idea of their theory is that, between the two extremes of the market and the government, there are many other public affairs that need to be governed. Since these subjects vary in terms of function, structure, external operating environment, and other features, we cannot apply a single approach on the supply of public goods to optimize the supply configuration of these services/goods [18]. Obviously, this theory emphasizes on the diversification of the structure of supply of public goods, advocating the provision of public good by the public sector, community organizations, the private sector, and community organizations and businesses, the introduction of competition mechanism pluralistic process to supply public goods in the past [19]. Additionally, it recommends network diversification and governance structure that overcomes the limitations of community governance by expanding the scope of the government. Finally, the citizen is considered the main focus of positive interaction with the private and public sectors to improve the efficiency of management of public affairs and increase the efficiency and ease pressure on governance.

In the past, our primary health care system has shown (1) a lack of system integration, integrated health system of external forces to support health service system deficiencies, overall development and coordination, collaboration, and integration of concepts; (2) isolated government action; (3) development of policies that lack complementarity; (4) inability to fully meet the different levels of needs of the masses; (5) inability to mobilize the enthusiasm of the masses and the main parties; and (6) poor efficiency [20]. The polycentric governance utilized for the implementation of community health management in Chinese medicine provides a new theoretical basis. The masses should demand the formation of a government-led system that involves citizen participation, multi-party cooperation of social organizations, innovation to build converged multi-center decision-making mechanisms, delivery mechanisms, and monitoring and evaluation mechanisms, as well as the characteristics of the Chinese medicine community health management model. In particular, attention needs to be focused on the mobilization of the enthusiasm of the masses and fostering community self-health management organizations. This will not only help reduce the burden on the government and help establish a community-oriented, flexible, and efficient operation mechanism, but will also be conducive to innovation and the expansion of service forms to meet the diverse needs of the residents.

The key characteristic of Chinese medicine community health management is that the system design is based on the utilization of community health management resources to optimize the allocation of the basic requirements of the Chinese medicine service and technology providers to meet the health demands of the masses; to encourage government organizations, health care institutions, universities, social organizations, enterprises, and residents to participate in cooperative governance; to promote the formation of the government and supplement the market; to foster the complementary synergistic effect of innovative operational mechanisms; and to apply Chinese medicine community health management for sustainable development.

\section{Conclusion}

In short, the use of Chinese medicine in community health management should be our national agenda, and a community health management system should be developed to take advantage of the characteristics of Chinese medicine. Established in the Community Governance government, social organizations, businesses, residents and other diversified system mode characteristic of Chinese medicine community to participate in health management and to improve the investment mechanism, interest distribution mechanism, and incentive and restraint mechanisms, to eventually improve the health of community residents in China.

\section{Acknowledgments}

The study was supported by the National Social Science Foundation of major projects funded project (12 \& ZD114) \& the National Social Science Fund Project (11BGL090). We thank Wang-qi for contributions to study design; Zhang-yan for contributions to study management; Zhang-wei for checking the Article's language and style.

\section{References}

[1] Lin Wei. On the development of community health services and their countermeasures [J]. Practical Preventive Medicine, 2011, 18(8): 1596-1598.

[2] Huang Yi-xiang. Health Management: Concept Definition and Model [J]. Journal of Wuhan University: Philosophy and Social Sciences, 2011, 64(6): 66-71.

[3] Huang Yi-xiang, Li Jiang-fan. Changes in demand for health services and medical paradigm shift [J]. Academic Journal of Zhongzhou, 2010, 32(1): 114-119.

[4] Larry SC, Kenneth RP. Population health management as a strategy for creation of optimal healing environments in worksite and corporate setting [J]. Journal of Alternative and Complementary Medicine, 2004, 10 (S1): 127-140.

[5] Chen Jun-shi, Huang Jian-shi. Health Management Division [M]. Beijing: China Union Medical University Press, 2007: 12.

[6] Edingtond $\mathrm{W}$, Louis $\mathrm{Y}, \mathrm{Ku}$ Kull, et al. Recent trends in the development of health management [J]. Health Management Research, 2001, 76 (103): 140-147.

[7] Zhu You-yuan, Zhao Ying, Pan Yi-hui, et al. Research and Practice of Community Health Service Management Model [J]. Chinese General Practice, 2012, 15 (7): 2202.

[8] State Administration of Traditional Chinese Medicine. Chinese Medicine Hospital of Traditional Chinese Culture Construction Guide [R]. Beijing: State Administration of Traditional Chinese Medicine, 2009. 
[9] Chen Li-yun, Yan Shi-yun. "He" the pursuit: traditional Chinese medicine treatment Philosophical Perspective [J]. Journal of East China Normal University (Philosophy and Social Sciences), 2011, 57(2): 29-36.

[10] Zheng Xiao-hong. Core value system of Chinese and Western cultures and universal values [J]. Chinese Journal of Basic Medicine in Traditional Chinese Medicine, 2012, 18(1): 108-109.

[11] Jin Qi, Wang Qi. On "Preventive Treatment" in Traditional Chinese Medicine [J]. Journal of Beijing University of Traditional Chinese Medicine, 2007, 30 (11): 725-727.

[12] Qu Bao-ping, Li Wen-lian. Talking about the role of Chinese Medicine in Community Health Service [J]. Gansu Journal of Traditional Chinese Medicine, 2008, 21(11): 80-81.

[13] Huang He. "Along the Way" in Public Perspective [J]. World Economics and Politics, 2015(6): 138-160.

[14] Li Bing, Jiang Ji-yu, Bi Yu-jian. Government rural public goods supply behavior and lack of institutional arrangements [J]. Productivity Research, 2007, 22 (16): 19-21.
[15] Tan Yu. Multiple polycentric governance theory and supply of affordable housing [J]. Urban Problems, 2012, 31(12): 63-66.

[16] Xu Xuan-guo, Yang Jun, Xu Yong-xiang. Government procurement of public services to the theoretical spectrum and beyond --- to Neoinstitutionalism Perspective [J]. Study and Practice, 2014(10): 92-101.

[17] Li Ping-yuan. On the applicability and limitations Ostrom polycentric governance theory - based government, market and society Governance of Pluralism perspective [J]. Tribune of Study, 2014(5): 50.

[18] Shang Hai-tao. Public goods supply diversification in its potential problems and efficiency of public view [J]. Qinghai Social Sciences, 2010, 31(5): 31-36.

[19] Chen Yan-min. Polycentric governance theory: a theory of the public system of things autonomous governance [J]. Tribune of Social Sciences in Xinjiang, 2007, 19(3): 35-38.

[20] Shi Ming-li. Status quo and institutional arrangement of primary health care system based on integrated perspective in China [J]. Chinese Journal of Health Policy, 2012, 5 (9): 28-33. 